\title{
Organização Espacial na Percepção Visual de Luminosidade ${ }^{1}$
}

\author{
Alexandre José Loureiro Ribeiro \\ Wânia Cristina de Souza² \\ Universidade de Brasília
}

\begin{abstract}
RESUMO - Este estudo analisou a influência de variações físicas dos estímulos, com base na organização espacial de figurafundo criada pela associação dos efeitos ilusórios de contraste simultâneo de luminosidade e de contornos subjetivos. A cada participante, no total de 64, foram apresentadas 160 matrizes de escolha, cada uma composta de um estímulo modelo e quatro estímulos de comparação, devendo ser identificado qual dos quatro estímulos de comparação correspondia ao estímulo modelo. A diferença significativa entre as médias de ajuste visual verificadas para a condição de contorno subjetivo médio e para a condição controle (sem contorno) mostrou que a formação clássica de contornos subjetivos de Kanizsa, quando associada ao efeito de contraste simultâneo de luminosidade, influenciou a percepção de luminosidade dos participantes.
\end{abstract}

Palavras-chave: percepção visual de luminosidade; contraste simultâneo de luminosidade; contornos subjetivos; organização espacial; percepção figura-fundo.

\section{Spatial Organization in Visual Perception of Lightness}

\begin{abstract}
This study investigated the influence of physical variations of stimuli, based on the spatial organization of figure-ground, which is created by the association of illusory effects of simultaneous lightness contrast and subjective contours. One hundred and sixty matrixes were presented to each participant, among a total of 64. Each matrix was composed of one sample stimulus and four comparison stimuli, and the task was to identify which of the four comparison stimuli corresponded to the sample stimulus. The significant difference between the averages of visual adjustment that were verified for the medium subjective contour and for the control condition (without contour) showed that the classical formation of Kanizsa subjective contours influenced visual perception when associated with the effect of simultaneous lightness contrast.
\end{abstract}

Keywords: visual perception of lightness; simultaneous lightness contrast; subjective contours; spatial organization; figureground perception.

O efeito do contraste simultâneo de luminosidade, efeito no qual uma região parece mais clara quando observada contra um fundo escuro e mais escura quando observado contra um fundo claro, tem intrigado filósofos e cientistas por mais de dois milênios, permanecendo até hoje sem um consenso a questão de porque exatamente isso ocorre. Segundo Sabra (1978), a questão, inicialmente abordada pelo filósofo grego Aristóteles (384 - 322 a.C.), passando pelo pouco conhecido filósofo árabe da Idade Média, Ibn Al-Haytham (965 a.C. - 1040 d.C.), também conhecido como Alhazen, em seguida pelo célebre físico e fisiologista inglês Isaac Newton (1642 - 1727), culminando nas abordagens experimentais dos físicos e fisiologistas alemães Hermann von Helmholtz (1866/1962), Ewald Hering (1874/1964) e David Katz (1935), permanece ainda hoje sem explicação.

Mesmo que atualmente uma quantidade significativa de pesquisas defendam a predominância sensorial no processamento da percepção de luminosidade (Anderson,1997;

1 Este relato de pesquisa baseia-se na Dissertação de Mestrado do primeiro autor, desenvolvida sob a orientação do segundo autor, com apoio financeiro da CAPES e do CNPq.

2 Autor para quem correspondências deverão ser enviadas: Universidade de Brasília, Instituto de Psicologia, Departamento de Processos Básicos. Brasília, DF. CEP 70910-900.E-mail: wcsouza@unb.br.
Blakeslee \& McCourt, 1999; Cataliotti \& Gilchrist, 1995; Kelly \& Grossberg, 2000; Ross \& Pessoa, 2000; Purves, Williams, Nundy \& Lotto, 2004; Todorovic, 1997), uma grande variedade de fenômenos visuais ligados a essa mesma submodalidade perceptual são diretamente relacionados a mecanismos complexos de processamento perceptual dos estímulos visuais. Entre estes, por exemplo, a análise da iluminação, da profundidade espacial, da transparência e do agrupamento entre os estímulos (Bell \& Badcock, 2008; Blakeslee \& McCourt, 2004; Blakeslee, Reetz \& McCourt, 2009; Buckley, Frisby \& Freeman, 1994; Kingdom, Blakeslee \& McCourt, 1997; Knill \& Kersten,1991; Paradiso, 2000; Perna \& Morrone, 2007; Purves, Shimpi \& Lotto, 1999; Schirillo \& Shevell, 1997; Wishart, Frisby \& Buckley, 1997).

Pesquisas recentes (Adelson, 1993, 2000; Gilchrist \& cols., 1999; Ichihara, Kitagawa \& Akutsu, 2007; Logvinenko, 1999, 2005) buscam identificar quais as principais características envolvidas na formação e na percepção visual do efeito de contraste simultâneo de luminosidade. Como identificado, por exemplo, por Bonato e Cataliotti (2000), a análise da organização espacial dos estímulos tem se mostrado elemento de fundamental importância na compreensão das características e dos processos relacionados à percepção visual do referido efeito. Nesse contexto, nosso estudo procurou identificar alterações na percepção 
visual de luminosidade em consequência de variações físicas dos estímulos, com base na organização espacial de figura-fundo criada pela associação dos efeitos ilusórios de contraste simultâneo de luminosidade e de contornos subjetivos.

Mais especificamente, o presente estudo investigou se os seguintes fatores podem ser responsáveis por variações na percepção do efeito de contraste simultâneo de luminosidade: (1) presença de formações ilusórias de contornos subjetivos de Kanizsa (1976); (2) variações físicas nos elementos formadores dos contornos subjetivos de Kanizsa; e (3) presença de elementos formadores de sobreposição aparente na configuração de contornos subjetivos de Kanizsa. A análise desses fatores foi feita em relação a duas condições distintas, denominadas mais e menos, cuja característica consistiu, respectivamente, no acréscimo ou decréscimo da gradação de cinza dos estímulos visuais de comparação.

\section{Método}

\section{Participantes}

A presente pesquisa, aprovada pelo Comitê de Ética em Pesquisa da Faculdade de Medicina da Universidade de Brasília, contou com a participação de 64 voluntários (16 homens e 48 mulheres), com idade variável entre 18 a 23 anos, todos alunos regulares da UnB. Para a participação dos voluntários, foi exigido o preenchimento do cadastro de participação e o termo de consentimento livre e esclarecido de participação.

Os participantes foram divididos em dois grupos de 32 participantes, denominados de Grupo Mais e Grupo Menos. O Grupo Mais realizou o procedimento de escolha do estímulo de comparação correto dentre quatro estímulos de comparação que apresentavam variações crescentes nos valores de gradação de cinza. O Grupo Menos realizou o procedimento de escolha do estímulo de comparação correto dentre quatro estímulos de comparação que apresentavam variações decrescentes nos valores de gradação de cinza.
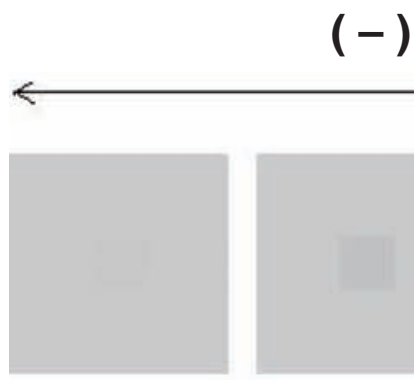

(a)

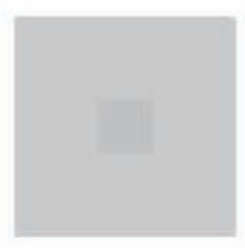

(b)

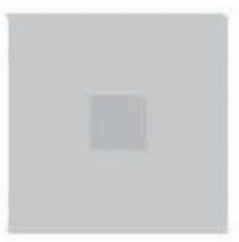

(c)

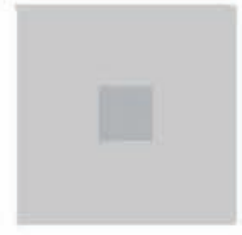

(d)

\section{Equipamento}

Em uma sala escura (3,85 m x 2,30 m x 2,40 m), um microcomputador foi utilizado para apresentar os estímulos visuais e coletar as respostas dos observadores por meio do programa MTS - Matching to Sample (Dube \& Hiris, 2001). O monitor foi posicionado de forma centralizada na altura dos olhos dos observadores e ajustado a $50 \mathrm{~cm}$ de distância dos mesmos com resolução de 800 x 600 pixels, frequência de atualização de imagem na tela no valor de $60 \mathrm{~Hz}$, e no modo de apresentação gráfica de milhões de cores.

\section{Estímulos}

Foram utilizados dois tipos de estímulos visuais: estímulos modelo e estímulos de comparação (Figura 1). Os estímulos modelo (Figura 1d) foram desenvolvidos a partir de 40 combinações de sobreposição de figura-fundo entre dois quadrados, um menor (41 pixels) e um maior (172 pixels), preenchidos com diferentes gradações de cinza. Os quadrados menores (figuras) foram preenchidos com gradações de $10 \%$, $20 \%, 30 \%, 40 \%, 50 \%, 60 \%, 70 \%, 80 \%$ e $90 \%$ de preto e localizados acima e ao centro dos quadrados maiores (fundos), preenchidos com gradações de $10 \%, 30 \%, 50 \%$, 70\% e $90 \%$ de preto. Os estímulos de comparação foram desenvolvidos a partir das mesmas 40 combinações de sobreposição de figura-fundo utilizadas na composição dos estímulos modelo, e utilizando-se variações, na ordem de 3\%, nos valores de gradação de cinza dos quadrados menores. Os estímulos de comparação apresentados aos participantes do Grupo Mais foram compostos de variações crescentes nos valores de gradação de cinza (Figuras 1e, 1f, 1g), e os apresentados ao Grupo Menos de variações decrescentes nos valores de gradação de cinza (Figuras 1a, 1b, 1c).

\section{Condições de comparação}

Cada conjunto de 40 combinações de sobreposição de figura-fundo dos dois quadrados preenchidos com diferentes gradações de cinza foi apresentado a partir de quatro condições de comparação (Figura 2): na condição de controle

Figura 1. Exemplos de estímulos utilizados na condição de controle: (d) representa o estímulo modelo criado a partir da combinação de sobreposição entre 2 quadrados, o menor preenchido com 30\% de preto e o maior preenchido com 20\% de preto; (a), (b) e (c) representam estímulos de comparação desenvolvidos a partir de variações decrescentes nos valores de preenchimento dos quadrados menores, diminuindo na ordem de 3\%; (e), (f) e (g) representam estímulos de comparação desenvolvidos a partir de variações crescentes nos valores de preenchimento dos quadrados menores, aumentando na ordem de 3\%. 
(ou condição sem contorno), os estímulos modelo foram comparados com estímulos de comparação sem a presença de qualquer formação de contorno subjetivo (Figura 2a); na condição de contorno fraco, os estímulos modelo foram comparados com estímulos de comparação inseridos numa formação simplificada de contorno subjetivo, derivada da composição clássica do quadrado de Kanizsa (Figura 2b); na condição de contorno médio, os estímulos modelo foram comparados com estímulos de comparação inseridos numa formação padrão de contorno subjetivo, diretamente relacionada à composição clássica do quadrado de Kanizsa (Figura 2c); e na condição de contorno forte, os estímulos modelo foram comparados com estímulos de comparação inseridos numa formação composta de contorno subjetivo, derivada da sobreposição aparente de elementos intermediários aos elementos presentes na composição clássica do quadrado de Kanizsa (Figura 2d).

\section{Delineamento - Matriz de escolha}

Os estímulos visuais utilizados no experimento foram apresentados no monitor do computador a partir de uma matriz de escolha composta por cinco quadrantes em forma de cruz, localizada sobre um fundo preto, sendo ambos os elementos (matriz e fundo) fornecidos pelo próprio programa MTS. A matriz de escolha apresentada em cada uma das 160 telas era composta de um estímulo modelo localizado no centro da matriz juntamente com quatro estímulos de comparação posicionados de forma aleatória dentro dos quatro quadrantes localizados ao redor do mesmo (um de gradação de cinza idêntica ao estímulo modelo e outros três de gradações de cinzas diferentes do estímulo modelo) (Figura 3).
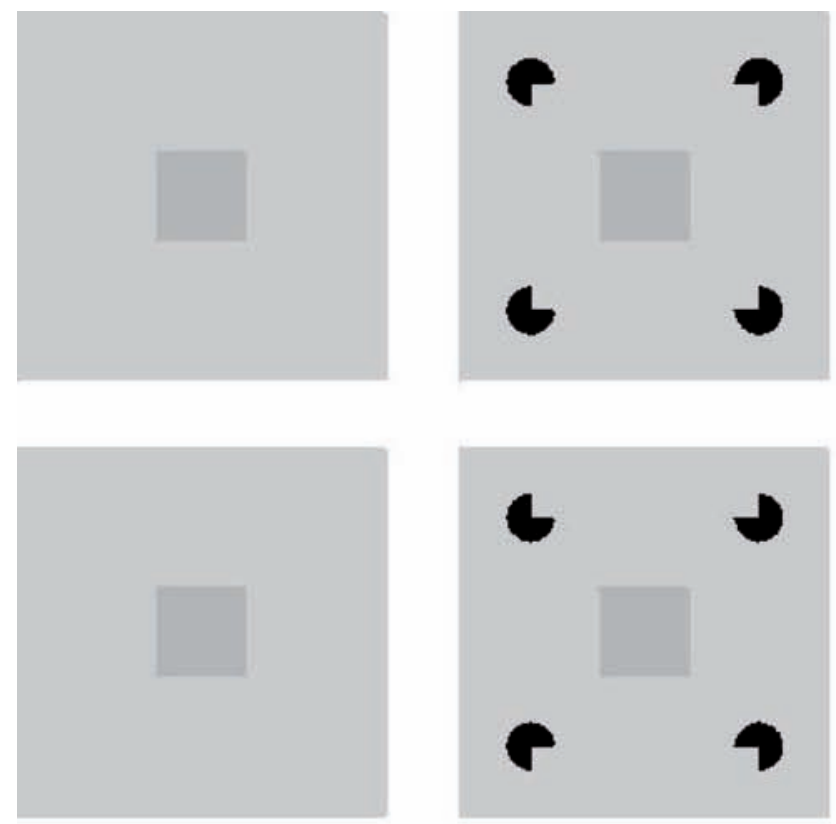

( a )

\section{Procedimento}

O procedimento experimental completo, para cada um dos 64 participantes, foi composto por um conjunto de 160 telas apresentadas de forma individual e aleatória entre os participantes (Figura 3). A tarefa do participante consistia em identificar, por meio de um clique com o cursor controlado pelo mouse em período de tempo livre, qual dos quatro estímulos de comparação possuía o quadrado menor preenchido com a mesma gradação de cinza do quadrado menor do estímulo modelo localizado no centro da matriz. Os participantes do Grupo Mais deveriam escolher o estímulo de comparação correto dentre os quatro estímulos de comparação que apresentavam variações crescentes nos valores de gradação de cinza (Figura 3a). Por sua vez, a tarefa dos participantes do Grupo Menos era identificar o estímulo de comparação correto dentre os quatro estímulos de comparação que apresentavam variações decrescentes nos valores de gradação de cinza (Figura 3b).

\section{Análise de dados}

Os dados armazenados pelo programa foram analisados com o objetivo de identificar possíveis padrões de percepção visual de luminosidade entre os participantes. A análise estatística da variabilidade das médias dos ajustes visuais apresentados para as quatro condições de comparação (sem contorno, contorno fraco, contorno médio e contorno forte) foi feita calculando-se uma ANOVA, e aplicando-se o teste Duncan para comparações posteriores. Comparações dos resultados obtidos em relação ao Grupo Mais e ao Grupo Menos foram realizadas por meio de um modelo de regressão
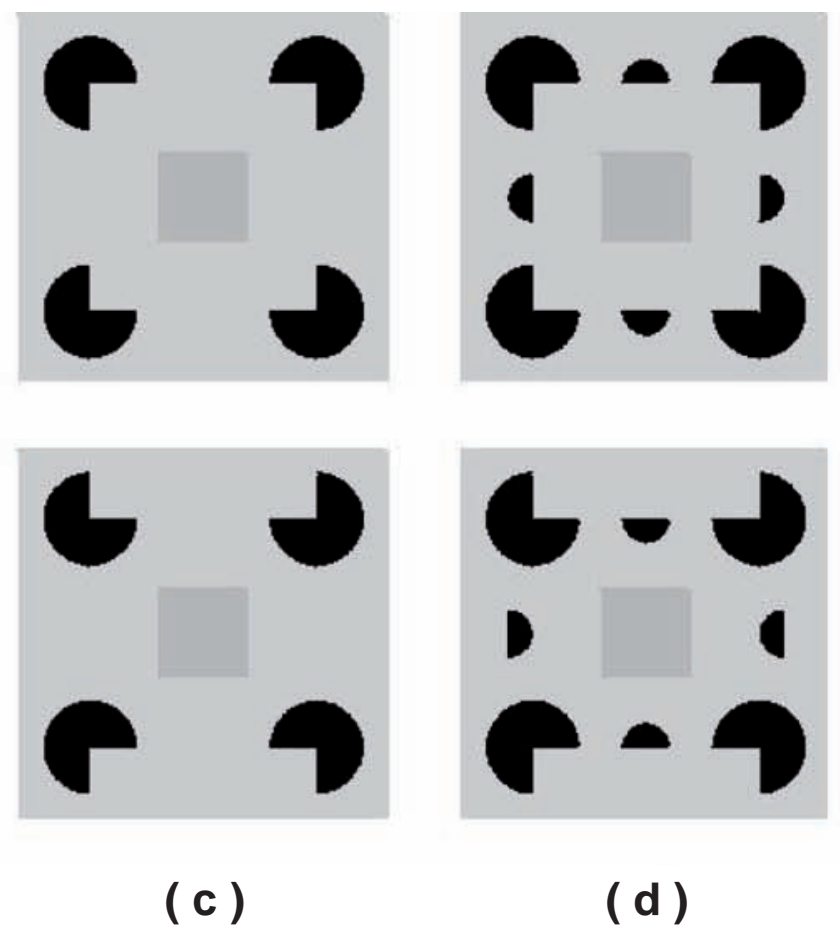

Figura 2. Exemplos de estímulos modelo (linha superior) e de seus respectivos estímulos de comparação (linha inferior) nas quatro condições de comparação: coluna (a), sem contorno; coluna (b), contorno fraco; coluna (c), contorno médio; e coluna (d), contorno forte. 


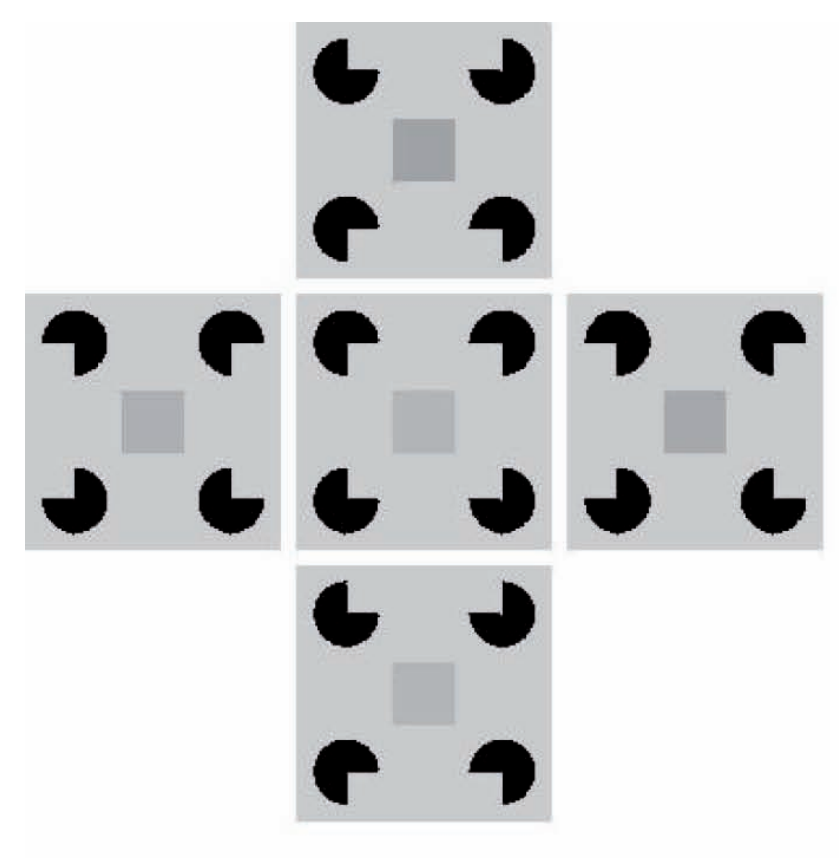

(a )

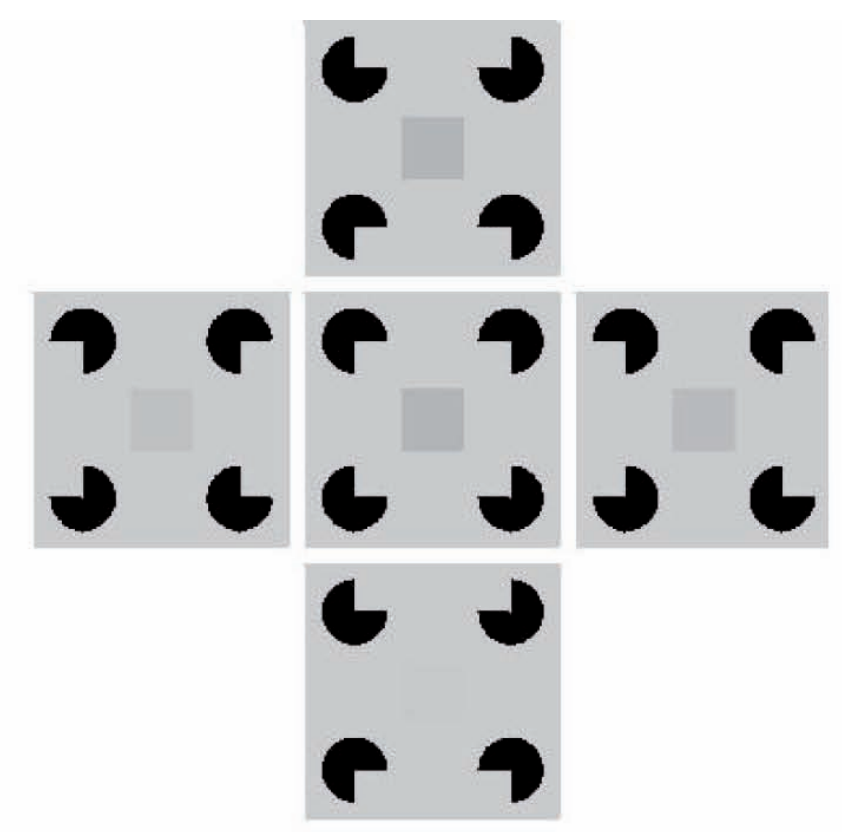

(b)

Figura 3. Exemplos da matriz de escolha, conforme as duas variações experimentais: (a) matriz com variações crescentes nos valores de gradação de cinza, apresentada aos participantes do Grupo Mais; e (b) matriz com variações decrescentes nos valores de gradação de cinza, apresentada aos participantes do Grupo Menos.

linear, para verificar em qual grupo os ajustes visuais foram maiores; e do teste de F-Snedecor, para analisar qual dos grupos apresentou maior variância de ajustes.

\section{Resultados}

As tabelas 1 e 2 mostram, respectivamente, as médias de ajuste visual dos participantes do Grupo Mais, e do Grupo Menos, após a realização do procedimento experimental.

A Tabela 1 mostra a comparação das médias das respostas dos participantes do Grupo Mais, expressas em porcentagem (\%), em relação às escolhas dos estímulos de comparação em cada uma das quatro condições de comparação (A, B, C e D). A maior média de ajuste visual $(1,76 \%)$ foi verificada para a condição de comparação $C$ (contorno médio), enquanto a menor média (1,49\%) correspondeu à condição de comparação A (sem contorno). Ajustes similares foram verificados para as condições B e D (1,68\% e 1,67\%, respectivamente). Pelo cálculo ANOVA das médias referentes às condições A, B, C e D, com 95\% de confiança, obteve-se os valores $F=3,06$ e $p=0,027$, indicando uma diferença estatisticamente significativa entre as médias de alguma(s) das quatro condições de comparação. Aplicando-se o teste Duncan para a comparação múltipla das médias referentes às condições $\mathrm{A}, \mathrm{B}, \mathrm{C}$ e D, foram obtidos dois grupos de testes distintos: Grupo I e Grupo II. A média de ajuste da condição $C$ (contorno médio) referiu-se exclusivamente ao Grupo I, enquanto a média de ajuste da condição A (sem contorno) referiu-se exclusivamente ao Grupo II. Por sua vez, as médias de ajuste das condições B e D foram verificadas indiscriminadamente para os grupos
I e II. A análise estatística pelo teste Duncan, portanto, demonstrou a diferença significativa entre as médias de ajuste visual referentes à condição $C$ (contorno médio) e à condição A (sem contorno).

Por sua vez, a Tabela 2 mostra a comparação das médias das respostas dos participantes do Grupo Menos, expressas em porcentagem (\%), em relação às escolhas dos estímulos de comparação em cada uma das quatro condições de comparação (A, B, C e D). As médias de ajuste visual de -1,28\% e - $1,08 \%$ foram verificadas para as condições de comparação D (contorno forte) e C (contorno médio), respectivamente. Ajustes intermediários foram verificados para as condições A e B (-1,21\% e -1,16\%, respectivamente). Pelo cálculo ANOVA das médias referentes às condições $\mathrm{A}, \mathrm{B}, \mathrm{C}$ e D, com $95 \%$ de confiança, obteve-se os valores $\mathrm{F}=1,81$ e $\mathrm{p}=0,1434$. Aplicando-se o teste Duncan para a comparação múltipla das médias referentes às condições A, B, C e D, foram obtidos dois grupos de testes distintos: Grupo I e Grupo II. A média de ajuste da condição $C$ (contorno médio) referiu-se exclusivamente ao Grupo I, enquanto a média de ajuste da condição D (contorno forte) referiu-se exclusivamente ao Grupo II. Por sua vez, as médias de ajuste das condições A e B foram verificadas indiscriminadamente para os grupos I e II. Apesar de a análise pelo teste Duncan indicar uma diferença entre as médias de ajuste visual referentes à condição C (contorno médio) e D (contorno forte), a diferença não é estatisticamente significativa, conforme indicado por $\mathrm{p}>0,05$ (ANOVA).

Procedendo-se a uma análise comparativa entre os resultados referentes ao Grupo Mais e ao Grupo Menos, foi utilizado um modelo de regressão linear, para verificar em qual grupo os ajustes visuais foram maiores. A 
Tabela 1. Médias de ajuste visual dos participantes do Grupo Mais, juntamente com os valores parciais e totais do erro padrão e do intervalo de confiança de 95\% das referidas médias em cada uma das quatro condições de comparação (A, sem contorno; B, contorno fraco; C, contorno médio; e D, contorno forte).

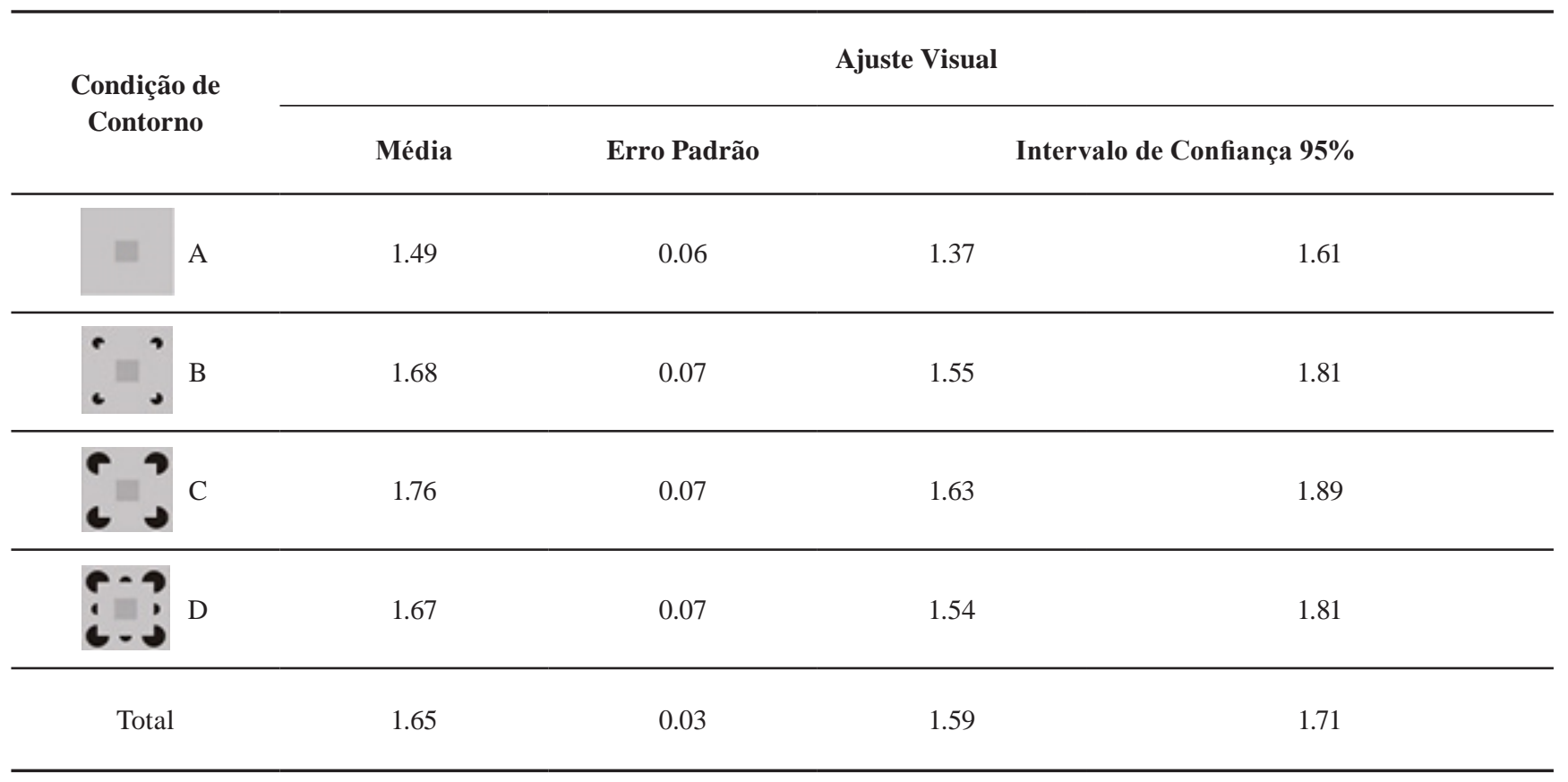

relação matemática linear descrita como [Percentual de ajuste $=1,18+0,47 *$ Grupo] calculou o ajuste de $1,65 \%$ para o Grupo Mais, e de $1,18 \%$ para o Grupo Menos, indicando que o ajuste foi maior no Grupo Mais. Adicionalmente, o teste de F-Snedecor foi utilizado para analisar qual dos grupos apresentou maior variância de ajustes. $\mathrm{O}$ teste calculou os valores $\mathrm{F}=1,12$ e $\mathrm{p}<0,0001$, bem como forneceu 5,53\% de variância para o Grupo Mais e 4,92\% de variância para o Grupo Menos, demonstrando que as variâncias referentes aos dois grupos foram estatisticamente significativas.

\section{Discussão}

Os valores de média de ajuste visual apresentados pelos participantes do Grupo Mais e do Grupo Menos ilustram as variações na percepção de luminosidade do efeito de contraste simultâneo de luminosidade criadas pela presença e pela variação física dos elementos formadores de contornos subjetivos e de sobreposição aparente.

Quanto às respostas dos participantes do Grupo Mais, a diferença estatisticamente significativa entre as médias de

Tabela 2. Médias de ajuste visual dos participantes do Grupo Menos, juntamente com os valores parciais e totais do erro padrão e do intervalo de confiança de 95\% das referidas médias em cada uma das quatro condições de comparação (A, sem contorno; B, contorno fraco; C, contorno médio; e D, contorno forte).

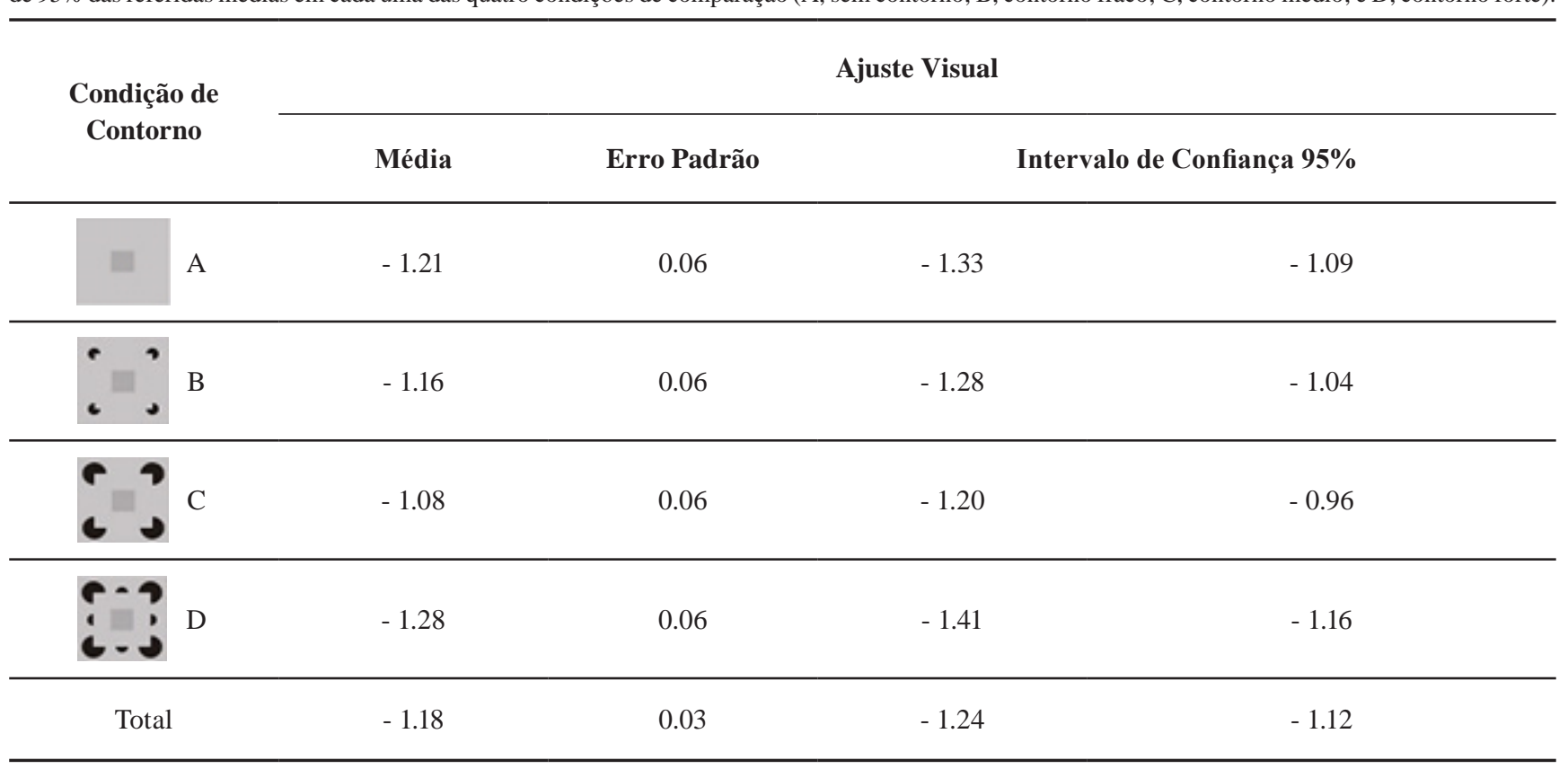


ajuste visual referentes às condições de comparação $\mathrm{A}$ (sem contorno) e C (contorno médio) (Tabela 1) demonstrou que a superfície ilusória criada pela formação de contorno subjetivo de Kanizsa (condição de comparação C) foi percebida como sendo mais luminosa do que o quadrado de fundo no qual estava inserida. Semelhante ao que ocorre no efeito de contraste simultâneo de luminosidade, onde uma região parece mais escura quando observada sobre um fundo mais claro, essa alteração perceptual parece ter resultado de uma nova organização espacial entre os estímulos visuais, criada pela relação de figura-fundo entre o quadrado menor e a superfície ilusória de contorno subjetivo. (Kingdom, 1997; Rock \& Palmer, 1990).

Por sua vez, os resultados indistintos obtidos em relação às médias de ajuste visual para as condições de comparação $\mathrm{B}$ (contorno fraco) e D (contorno forte) (Tabela 1), mostraram que variações físicas criadas pela alteração dos elementos estruturais e pela presença de estímulos formadores de sobreposição aparente não influenciaram significativamente novas variações ilusórias.

Pela comparação das médias de ajustes visuais apresentados pelos participantes do Grupo Mais e do Grupo Menos, verificou-se que padrões de variação apresentados em um grupo acabam por complementar os padrões de variação apresentados pelo outro grupo. $\mathrm{O}$ aumento na ilusão de luminosidade do Grupo Mais, chegando ao valor máximo de 1,76\% na condição de comparação C (contorno médio) (Tabela 1), e a equivalente diminuição na ilusão de luminosidade do Grupo Menos, chegando ao valor mínimo de -1,08\% também na condição de comparação C (Tabela 2), indicam que a presença da formação do contorno subjetivo referente à composição clássica do quadrado de Kanizsa alterou diretamente a percepção de luminosidade dos participantes de ambos os grupos, aumentando a ilusão de luminosidade dos participantes do Grupo Mais e diminuindo o mesmo efeito, embora de forma não significativa, nos participantes do Grupo Menos.

Tais resultados indicam que a análise perceptual do contexto visual criado pela organização espacial de figura-fundo, consequente da relação direta entre a superfície luminosa percebida a partir da formação ilusória de contornos subjetivos e elementos internos a essa formação, fornece elementos visuais significativos para a interpretação da luminosidade da cena analisada pelo observador. Dessa forma, o sistema visual aparentemente utiliza-se de associações complexas entre as características físicas dos estímulos visuais, como por exemplo, o agrupamento de elementos, a identificação de planos espaciais e o destaque visual das figuras analisadas em relação ao fundo no qual se encontram, para determinar a forma como os mesmos serão percebidos e interpretados.

Em outras palavras, os resultados apresentados indicam que não apenas os fenômenos ilusórios baseados em características físicas bem definidas, como, por exemplo, a iluminação ambiente e a definição do contorno, são responsáveis por variações ópticas na percepção de seus observadores, mas também podem ser incluídos a esse grupo de variações, efeitos visuais complexos diretamente associados à organização espacial de figura-fundo criada a partir da formação de contornos subjetivos.
O presente estudo apresenta características comuns com pesquisas anteriores relacionadas à influência da organização espacial dos estímulos na percepção de luminosidade. Gilchrist (1977) verificou que a percepção de gradação de cinza depende diretamente da relação de luminosidade entre superfícies que se encontram no mesmo plano, e não da relação de superfícies meramente adjacentes na imagem formada na retina, sugerindo que a percepção espacial de profundidade precederia a percepção de luminosidade. Aparentemente, teorias baseadas exclusivamente no processamento sensorial da informação visual, portanto, não explicariam de forma satisfatória variações perceptuais resultantes de arranjos espaciais articulados.

Em um estudo sobre a relação entre a organização perceptual e a análise visual de luminosidade, Adelson (1993) relatou que mudanças na interpretação perceptual das formas tridimensionais de uma superfície produziam fortes efeitos na percepção de luminosidade da mesma. Foi sugerido que as junções entre os diferentes planos da superfície representava um elemento importante na determinação da organização perceptual e na consequente delimitação de tais efeitos. Posteriormente, verificou que elementos complexos resultantes da organização espacial de agrupamento, tais como as características físicas de proximidade e de similaridade, também poderiam estar associados à influência exercida pelas variações de junções na percepção visual de luminosidade (Adelson, 2000).

Os efeitos da relação de figura-fundo, da área percebida e da forma do estímulo na definição do limiar visual de luminosidade, foram investigados por Bonato e Cataliotti (2000). Esses autores verificaram que estímulos visuais percebidos como figuras, relativamente menores em área ou que não se agrupassem com outros elementos presentes no campo visual, foram percebidos como sendo mais luminosos pelos participantes da pesquisa do que efetivamente eram. Portanto, não apenas a percepção de luminosidade, mas também o limiar visual de luminosidade era influenciado pela organização espacial dos estímulos no campo visual, não se relacionando tal processo apenas a mecanismos sensoriais ocorridos na retina.

Em conclusão, nossos resultados mostram que a relação espacial de figura-fundo, criada a partir da formação ilusória de contornos subjetivos, pode influenciar significativamente a percepção visual de luminosidade, assim como ocorre com as percepções de tamanho, profundidade e de agrupamento. Corroboram-se também as evidências de que teorias baseadas exclusivamente no processamento sensorial da informação visual não explicam de forma completa a grande variedade de fenômenos e ilusões criadas a partir de formações complexas desses estímulos.

\section{Referências}

Adelson, E. (1993). Perceptual organization and the judgement of brightness. Science, 262, 2042-2044.

Adelson, E. (2000). Lightness perception and lightness illusions. Em M. Gazzaniga (Org.), The new cognitive neuroscience (pp. 339-351). Cambridge: MIT. 
Anderson, B. (1997). A theory of illusory lightness and transparency in monocular and binocular images: The role of contour junctions. Perception, 26, 419-453.

Bell, J., \& Badcock, D. R. (2008). Luminance and contrast cues are integrated in global shape detection with contours. Vision Research, 48, 2336-2344.

Blakeslee, B., \& McCourt, M. E. (1999). A multiscale spatial filtering account of the White effect, simultaneous brightness contrast and grating induction. Vision Research, 39, 4361-4377.

Blakeslee, B., \& McCourt, M. E. (2004). A unified theory of brightness contrast and assimilation incorporating oriented multiscale spatial filtering and contrast normalization. Vision Research, 44, 2483-2503.

Blakeslee, B., Reetz, D., \& McCourt, M. E. (2009). Spatial filtering versus anchoring accounts of brightness/lightness perception in staircase and simultaneous brightness/lightness contrast stimuli. Journal of Vision, 26, 1-17.

Bonato, F., \& Cataliotti, J. (2000). The effects of figure-ground, perceived area, and target saliency on the luminosity threshold. Perception \& Psychophysics, 62, 341-349.

Buckley, D., Frisby, J., \& Freeman, J. (1994). Lightness perception can be affected by surface curvature from stereopsis. Perception, 23, 869-881.

Cataliotti, J., \& Gilchrist, A. (1995). Local and global processes in surface lightness perception. Percept Psychophy, 52, 125-135.

Dube, W. V., \& Hiris, E. J. (1996). MTS v 11.6 [Software for research and education]. Waltham, MA: E. K. Shriver Center for Mental Retardation.

Gilchrist, A. (1977). Perceived lightness depends on perceived spatial arrangement. Science, 195, 185-187.

Gilchrist, A., Kossyfidis, C., Bonato, F., Agostini, T., Cataliotti, J., Li, X., Spehar, B., \& Szura, J. (1999). An anchoring theory of lightness perception. Psychology Review, 106, 795-834.

Helmholtz, H. (1962). Treatise on physiological optics. New York: Dover. (Trabalho original publicado em 1866)

Hering, E. (1964). Outlines of a theory of the light sense. Cambridge: Harvard University. (Trabalho original publicado em 1874)

Ichihara, S., Kitagawa, N., \& Akutsu, H. (2007). Contrast and depth perception: Effects of texture contrast and area contrast. Perception, 36, 658-695.

Kanizsa, G. (1976). Subjective contours. Scientific American, 234, 48-52.

Katz, D. (1935). The world of colour. Londres: Kegan Paul, Trench,Trubner \& Co.

Kelly, F., \& Grossberg, S. (2000). Neural dynamics of 3-D surface perception: Figure-ground separation and lightness perception. Perception \& Psychophysics, 62, 1596-1618.
Kingdom, F., Blakeslee, B., \& McCourt, M. (1997). Brightness with and without perceived transparency: When does it make a difference? Perception, 26, 493-506.

Knill, D., \& Kersten, D. (1991). Apparent surface curvature affects lightness perception. Nature, 351, 228-230.

Logvinenko, A. (1999). Lightness induction revisited. Perception, 28, 803-816.

Logvinenko, A. (2005). Does luminance contrast determine lightness? Spatial Vision, 18, 337-345.

Paradiso, M. (2000). Visual neuroscience: Illuminating the dark corners. Current Biology, 10, R15-R18.

Perna, A., \& Morrone, M. C. (2007). The lowest spatial frequency channel determines brightness perception. Vision Research, 47, 1282-1291.

Purves, D., Shimpi, A., \& Lotto, R. (1999). An empirical explanation of the Cornsweet effect. The Journal of Neuroscience, 19, 8542-8551.

Purves, D., Williams, S. M., Nundy, S., \& Lotte, R. B. (2004). Perceiving the intensity of light. Psychological Review, 111, 142158.

Ross, W., \& Pessoa, L. (2000). Lightness from contrast: A selective integration model. Perception \& Psychophysics, 62, 1160-1181.

Rock, D., \& Palmer, S. (1990). The legancy of Gestalt psychology. Scientific American, 263, 84-90.

Sabra, A. (1978). Sensation and inference in Alhazen's theory of visual perception. Em P. Machamer \& R. Turnbull (Orgs.), Studies in perception: Interrelations in the history of philosophy and science (pp. 160-161). Columbus: Ohio State University.

Schirillo, J., \& Shevell, S. (1997). An account of brightness in complex scenes based on inferred illumination. Perception, 26 , 507-518.

Todorovic, D. (1997). Lightness and junctions. Perception, 26, 379-394.

Wishart, K., Frisby, J, \& Buckley, D. (1997). The role of 3-D surface slope in a lightness/brightness effect. Vision Research, 37, 467-473. 